\title{
The spectral lines of the charge 2 axially symmetric monopole
}

To cite this article: Anthony Small 2002 J. Phys. A: Math. Gen. 355763

View the article online for updates and enhancements. 


\title{
The spectral lines of the charge 2 axially symmetric monopole
}

\author{
Anthony Small \\ Department of Mathematics, National University of Ireland, Maynooth, Co. Kildare, \\ Republic of Ireland \\ E-mail: asmall@maths.may.ie
}

Received 1 March 2002, in final form 17 May 2002

Published 28 June 2002

Online at stacks.iop.org/JPhysA/35/5763

\begin{abstract}
The configuration of spectral lines of the axially symmetric charge 2 monopole is described.

PACS numbers: $14.80 . \mathrm{Hv}, 02.40 .-\mathrm{k}$

Mathematics Subject Classification: 53C07, 81T13
\end{abstract}

\section{Introduction}

$S U(2)$ BPS-monopoles are certain static particle-like solitons in Yang-Mills-Higgs theory in the Prasad-Sommerfeld limit on $\mathbb{R}^{3}$. Finiteness of the energy means that the Higgs field at infinity determines a winding number $k$, which (in suitable units) is the total magnetic charge of the monopole. The Bogomolny bound says that the energy of a charge $k$ monopole is bounded below by $4 \pi|k|$. For a fixed charge $k>0$, the minima of the energy are, by definition, the BPS-monopoles of charge $k$. Henceforth by 'monopole' we mean SU(2) BPS-monopole. See $[1,3,6]$ for further details and references.

These minima are solutions of the first order Bogomolny equation. That equation may, by means of a twistor transform, be reinterpreted as the holomorphicity of an auxiliary rank 2 complex vector bundle over $\mathbb{T}$, the space of all oriented lines in $\mathbb{R}^{3}$. Finiteness of the energy means that the bundle, and thus the monopole, is determined by an auxiliary algebraic curve in $\mathbb{T}$. This is the so-called spectral curve of the monopole; it parameterizes the monopole's spectral lines. See [1-3] for further details.

In general, it is hard to read the details of a monopole's structure from its spectral curve. For example, though it is easy to see the total energy, since the spectral curve of a charge $k$ monopole is a $k$-fold branched cover of $\mathbb{P}_{1}$ in $\mathbb{T}$, for plots of the energy density in $\mathbb{R}^{3}$, we rely on numerical methods, even when $k=2$.

It is natural to explore the relationship between the geometry of the configuration of the monopole's spectral lines and the energy density. It is easy to argue heuristically that spectral lines pass through regions where the Higgs field 'changes rapidly' and hence they go through 
the monopole particles. However, this picture is harder to understand for solutions that are not 'well-separated'. We now review this briefly in the charge 2 case.

First we recall that $\mathbb{T}$ may be identified with the total space of the holomorphic tangent bundle of $\mathbb{P}_{1}$, which may also be viewed as the space of all affine null planes in $\mathbb{C}^{3} \cong \mathrm{H}^{0}\left(\mathbb{P}_{1}, \mathcal{O}(\mathbb{T})\right)$. For a point $\underline{x}$ in $\mathbb{R}^{3}$, the corresponding global section of $\mathbb{T}$, which is given by $\sigma_{\underline{x}}(\zeta)=\underline{x} \cdot\left(1-\zeta^{2}, \mathrm{i}\left(1+\bar{\zeta}^{2}\right),-2 \zeta\right) \mathrm{d} / \mathrm{d} \zeta$, parameterizes the affine null planes through $\underline{x}$, each of which cuts out a line in $\mathbb{R}^{3}$ and endows it with a direction. Thus the real section may be viewed as parameterizing the two sphere of oriented lines that pass through $\underline{x}$. (For a point $\underline{z} \in \mathbb{C}^{3}$, away from $\mathbb{R}^{3}$, the picture of the configuration of lines in $\mathbb{R}^{3}$ determined by intersection with the affine null planes through $\underline{z}$ is not quite so simple.)

When $k=2$, non-axially symmetric monopoles have elliptic spectral curves. In the limit as the monopole 'separates', the spectral curve approaches degeneration to the pair of real sections corresponding to the two points $m$ and $m^{\prime}$, at distance $K / 2$, from the origin on the Higgs axis (where $K$ denotes Legendre's complete elliptic integral of the first kind), see chapter 7 of [1] for further details. This means that as $k \longrightarrow 1$, the configuration of spectral lines in $\mathbb{R}^{3}$ approximates the two 'stars' comprising all the lines through $m$ and $m^{\prime}$. It is known (numerically) that the energy density concentrates at these points.

Recall that the spectral curve of the charge 1 monopole at the origin is the zero section; this gives all the lines through $\underline{0}$. So in terms of the spectral lines, the 'well-separated' charge 2 monopole resembles a superposition of charge 1 monopoles located at $m$ and $m^{\prime}$. However, this picture does not persist when the 'particles' are not far apart. In particular, in the 'collision state', the spectral curve of a charge 2 axially symmetric monopole, is given, after a rotation of $\mathbb{R}^{3}$, by $S: \eta^{2}+\left(\frac{\pi}{2}\right)^{2} \zeta^{2}=0$, see [2]. So it reduces to the two sections given by: $\eta= \pm \mathrm{i} \frac{\pi}{2} \zeta$. These parameterize the two 'stars' of 'spectral affine null planes' through $(0,0, \pm \mathrm{i} \pi / 4) \in \mathbb{C}^{3}$.

As for the energy, it is known numerically in the axially symmetric case to be concentrated close to the monopole's harmonic locus, i.e. points in $\mathbb{R}^{3}$ where the two spectral lines are orthogonal; this is a toroidal ring, cf [3].

In [5], it is shown that the distribution of energy is elucidated by the behaviour of the measure induced on the spectral curve by the projection map to $\mathbb{P}_{1}$. This requires further study when the monopoles are close. It is useful to set down any precise quantitative information we have about the axially symmetric solution; in this note we set ourselves the simple task of describing the configuration of its spectral lines. In particular, we give an explicit description of the harmonic locus.

\section{The lines}

A point $(\zeta, \eta \mathrm{d} / \mathrm{d} \zeta) \in \mathbb{T}$, determines an oriented line in $\mathbb{R}^{3}$, whose distance from the origin is $|\eta| /\left(1+|\zeta|^{2}\right)$. Writing $\zeta=r \mathrm{e}^{\mathrm{i} \theta}$, for lines determined by $\eta= \pm \mathrm{i} \frac{\pi}{2} \zeta$, this distance is $\pi r / 2\left(1+r^{2}\right)$, which has maximum value $\pi / 4$, on the circle $r=1$.

Lemma. For the spectral curve $S$ :

(i) when $\zeta=\mathrm{e}^{\mathrm{i} \theta}$, the spectral lines lie in the $\left(x_{3}=0\right)$-plane.

(ii) The lines in the $\left(x_{3}=0\right)$-plane that are tangent to the circle $C$, given by $\left(x_{3}=0\right) \cap$ $\left(x_{1}^{2}+x_{2}^{2}=\pi^{2} / 16\right)$, are spectral lines.

Proof. For $\zeta=\mathrm{e}^{\mathrm{i} \theta}$, the associated spectral lines have equations given by:

$$
\underline{x} \cdot\left(1-\mathrm{e}^{2 \mathrm{i} \theta}, \mathrm{i}\left(1+\mathrm{e}^{2 \mathrm{i} \theta}\right),-2 \mathrm{e}^{\mathrm{i} \theta}\right)= \pm \frac{\mathrm{i} \pi}{2} \mathrm{e}^{\mathrm{i} \theta} .
$$

This is simply $\mathrm{i}\left(x_{1}, x_{2}\right) \cdot(-\sin \theta, \cos \theta)=x_{3} \pm \frac{\mathrm{i} \pi}{4}$. 


\section{Remarks.}

(i) Through each point on $C$ there is a unique spectral line; generically for a point in $\mathbb{R}^{3}$ there are two. This reflects the fact that $C$ is the real bitangent locus of $S$.

(ii) $C$ is the singular locus of the 'asymptotic Higgs field', see [4].

(iii) Any point on the $x_{3}$-axis, which is a spectral line, has only that spectral line through it.

\section{Proposition.}

(i) For a point $P$ in the $\left(x_{3}=0\right)$-plane outside $D$, the closed disc of radius $\pi / 4$, the two spectral lines through $P$ are the lines through $P$ that are tangent to $C$.

(ii) No spectral line on the $\left(x_{3}=0\right)$-plane passes into the interior of $D$.

(iii) Every spectral line in $\mathbb{R}^{3}$ intersects $D$.

Proof. (ii) and (iii) follow from (i). If either were not true it would mean that there was a point (in the $\left(x_{3}=0\right)$-plane) on at least three spectral lines, which is impossible.

Now consider the spectral lines which pass through the interior of $D$. The configuration of spectral lines in $\mathbb{R}^{3}$ enjoys circular symmetry about the $x_{3}$-axis, therefore it is sufficient to understand the behaviour of the spectral lines which pass through the line segment on the $x_{1}$-axis beween the origin and $x_{1}=\pi / 4$, which we call $L$.

\section{Proposition.}

(i) At the end points of $L$, the spectral lines coalesce: at $x_{1}=0$, the angle between the lines is 0 , while at $x_{1}=\pi / 4$, it is $\pi$.

(ii) At every point on $L$, the spectral lines lie in the plane parallel to the $\left(x_{1}=0\right)$-plane.

(iii) The closest point to the origin on any spectral line lies in the $\left(x_{3}=0\right)$-plane.

Proof. (i) is already clear. (ii) The directions of the spectral lines through $\left(x_{1}, 0,0\right)$ are given by solving $x_{1}\left(1-\zeta^{2}\right)= \pm \frac{\mathrm{i} \pi}{2} \zeta$, which gives, for $0<x_{1}<\pi / 4: \zeta= \pm \frac{\mathrm{i}}{4 x_{1}}\left(\pi \pm \sqrt{\pi^{2}-16 x_{1}^{2}}\right)$. Since $\zeta$ lies on the imaginary axis, the direction of the spectral line is perpendicular to the $x_{1}$-axis. (iii) is now clear too.

Remark. Note that the angle between the spectral lines at points on $L$ increases monotonically from 0 to $\pi$, as $x_{1}$ goes from 0 to $\pi / 4$.

\section{The harmonic locus}

In [3], the set of points in $\mathbb{R}^{3}$, where the spectral lines are orthogonal is considered for a monopole of charge 2 . The roots determined by the spectral lines through a point on this set are harmonically separated: accordingly we will refer to it as the harmonic locus of the monopole. Let $\mathcal{H}$ denote the harmonic locus of the reduced and centred axially symmetric monopole.

Proposition. $\mathcal{H}$ is the torus of revolution swept out by rotating the circle in the $\left(x_{1}, x_{3}\right)$-plane, of radius $\pi / 8 \sqrt{2}$, centred at $(3 \pi / 8 \sqrt{2}, 0,0)$, about the $x_{3}$-axis.

Proof. By axial symmetry it is sufficient to inspect $\mathcal{H} \cap\left(x_{2}=0\right)$. This means first writing down the roots of $x_{1} \zeta^{2}+\left(2 x_{3}+\mathrm{i} \pi / 2\right) \zeta-x_{1}=0$, and $x_{1} \zeta^{2}+\left(2 x_{3}-\mathrm{i} \pi / 2\right) \zeta-x_{1}=0$. Setting the appropriate cross ratio to -1 , gives the equation

$$
\pi^{4}+\pi^{2}\left(32 x_{3}^{2}-40 x_{1}^{2}\right)+256\left(x_{1}^{2}+x_{3}^{2}\right)^{2}=0
$$


which is equivalent to

$$
\left\{\left(x_{1}-\frac{3 \pi}{8 \sqrt{2}}\right)^{2}+x_{3}^{2}-\frac{\pi^{2}}{128}\right\}\left\{\left(x_{1}+\frac{3 \pi}{8 \sqrt{2}}\right)^{2}+x_{3}^{2}-\frac{\pi^{2}}{128}\right\}=0 .
$$

\section{Acknowledgments}

The author thanks the referees for their helpful comments. He also thanks the Mathematics Faculty of Southampton University, England, and the Mathematics Section of the ICTP, Trieste, Italy, for their generous hospitality during the preparation of this paper.

\section{References}

[1] Atiyah M F and Hitchin N J 1988 The Geometry and Dynamics of Magnetic Monopoles (Princeton: Princeton University Press)

[2] Hitchin N J 1982 Monopoles and geodesics Commun. Math. Phys. 83 579-602

[3] Hitchin N J 1987 Monopoles, Minimal Surfaces and Algebraic Curves (Montreal: Les Presses De L'Université De Montréal)

[4] Hurtubise J 1985 The asymptotic Higgs field of a monopole Commun. Math. Phys. 97 381-9

[5] Small A J 2002 Minimal surfaces from monopoles Preprint math.DG/0203223

[6] Sutcliffe P M 1997 BPS monopoles Int. J. Mod. Phys. A 12 4663-705 